\title{
Intramedullary screw fixation in proximal fifth-metatarsal fractures in sports: clinical and biomechanical analysis
}

\author{
André Leumann • Geert Pagenstert • Peter Fuhr • \\ Beat Hintermann • Victor Valderrabano
}

Received: 20 October 2007 / Published online: 2 August 2008

(C) Springer-Verlag 2008

\begin{abstract}
Introduction and purpose Intramedullary screw fixation (ISF) of proximal fifth-metatarsal fractures is known as first treatment option in young, sports active patients. No study analyzed functional and biomechanical outcome before. Hypothetically ISF leads to (1) a high bony union rate within 12 weeks, (2) normal hindfoot eversion strength, and (3) normal gait and plantar pressure distribution.

Methods Fourteen out of 22 patients were available for follow-up with an average follow-up of 42 months; clinical and radiological follow-up, and biomechanical evaluation by isometric muscular strength measurement (inversion, eversion strength) and dynamic pedobarography, comparing to the non-affected contralateral foot. Level of significance: 0.05 .

Results Subjective result: Excellent or good result in 14 patients, none fair or poor. AOFAS midfoot score: 100 points in 13 patients and 87 points in 1 patient. The same sports activity level (0-4) was reached in 13 out of 14 patients. Radiologic examination: consolidation after 6 weeks in
\end{abstract}

A. Leumann $\cdot$ V. Valderrabano $(\bowtie)$

Orthopaedic Department, Lower Extremity Orthopaedics,

University Hospital of Basel, Spitalstr. 21,

4031 Basel, Switzerland

e-mail: vvalderrabano@uhbs.ch

A. Leumann

e-mail: leumanna@uhbs.ch

G. Pagenstert · B. Hintermann

Orthopaedic Department,

Kantonsspital Liestal, Liestal, Switzerland

P. Fuhr

Neurologic Department,

University Hospital of Basel, Basel, Switzerland
9 patients and after 12 weeks in another 4 patients, one partial union. Average maximal eversion strength $59 \mathrm{~N}$ (ratio to the contralateral foot: 0.92 , not significant). Dynamic pedobarography showed ratios of $0.99-1.01$ to the contralateral side for ground reaction force, ground peak time, peak pressure and contact area (not significant).

Interpretation A very-high patient-satisfaction, a fast bony healing and complete return to sports were documented. Muscular strength measurement and dynamic pedobarography showed complete functional rehabilitation. Therefore, ISF in proximal fifth-metatarsal fractures can be recommended as a secure procedure.

Keywords Proximal fifth-metatarsal fracture · Intramedullary screw fixation · Biomechanics · Gait · Dynamic pedobarography

\section{Introduction}

Fractures of the proximal third of the fifth metatarsal (Fig. 1) are common among athletes. Particularly involved are soccer players [1] and basketball players [2]. Low et al. [1] found in college football players an incidence for Jones factures of $1.8 \%$. Since Sir Robert Jones 1902 [3], treatment of fractures of the proximal fifth-metatarsal remained controversial [4] aiming for fast osseous consolidation and complete and fast restoration of function.

The main concerns of functional impairment, one may think of eversion weakness because of damage of the Peroneus brevis tendon during the injury or the surgery and or the change of lever arm of the Peroneus brevis because of change of the position of the avulsed osseus-tendineous fragment (e.g. in avulsion type fractures). Secondly one may think of a change of gait patterns, either due to the 
Fig. 1 Classification of proximal fifth-metatarsal fractures. a Avulsion fracture of the tuberosity. b Jones fracture (metaphyseal fracture). c Proximal diaphyseal fracture
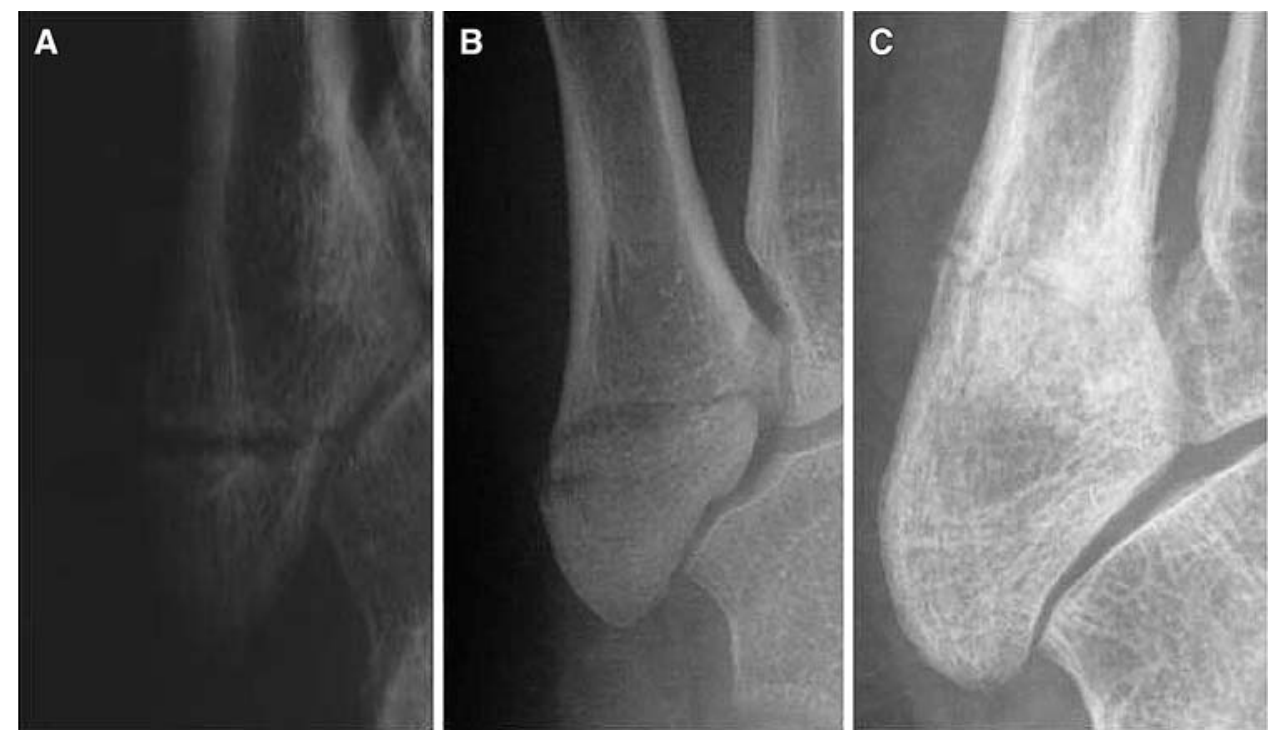

All subjects were free to participate and gave written informed consent. The study was carried out in accordance with the World Medical Association Declaration of Helsinki.

As surgical procedure, a short horizontal incision of the length of $2-3 \mathrm{~cm}$ is enough to access the situs (Fig. 2a). Following structures have to be considered: the sural nerve (runs just above the incision subcutaneously), the Peroneus brevis tendon (PBT) (This is the guiding structure), the Peroneus longus tendon (runs underneath the PBT turning around the os cuboideum in a hypomochlion-manner), and the Peroneus tertius tendon (from the dorsal side a small tendon may run facultatively into the operation situs inserting on the dorsal aspect of the fifth-metatarsal base) (Fig. 2b).

To pre-position the screw, a k-wire is inserted between the plantar fascia insertion and the PBT to optimize anatomical and biomechanical positioning and to minimize screwhead problems. Screw types may be cannulated, or in cases of a very-high stress demand, a non-cannulated screw may be chosen. Size of the screw varies between 4.0 and $6.5 \mathrm{~mm}$, a lag screw may enhance the interfragment compression. The largest possible screw size should be chosen. Alternatively in avulsion fractures with a too small fragment, a bicortical fixation with a smaller screw may be possible.

Full-weight bearing was achieved usually 2-4 weeks after the surgery depending on healing and subjective comfort. External stabilization is added, minimally with a stiffsole shoe or an ankle stabilizing shoe, maximally with a cast depending on patient's compliance. Rehabilitation training is started 2 weeks after surgery under physiotherapeutic support. If the fracture site is found to be stable and consolidated, free sport activity was allowed (Fig. 2c).

Clinical follow-up consisted of a standardized questionnaire: pre-trauma sports activity level (SAL) [5], rehabilitation 
Fig. 2 Surgical technique of intramedullary screw fixation. Intraoperative views of a 41-year-old male with an acute avulsion fracture of the fifthmetatarsal base. a Landmarks and skin incision. b K-wire positioning between peroneal brevis tendon and plantar fasial insertion. c X-ray follow-up 6 weeks postoperative
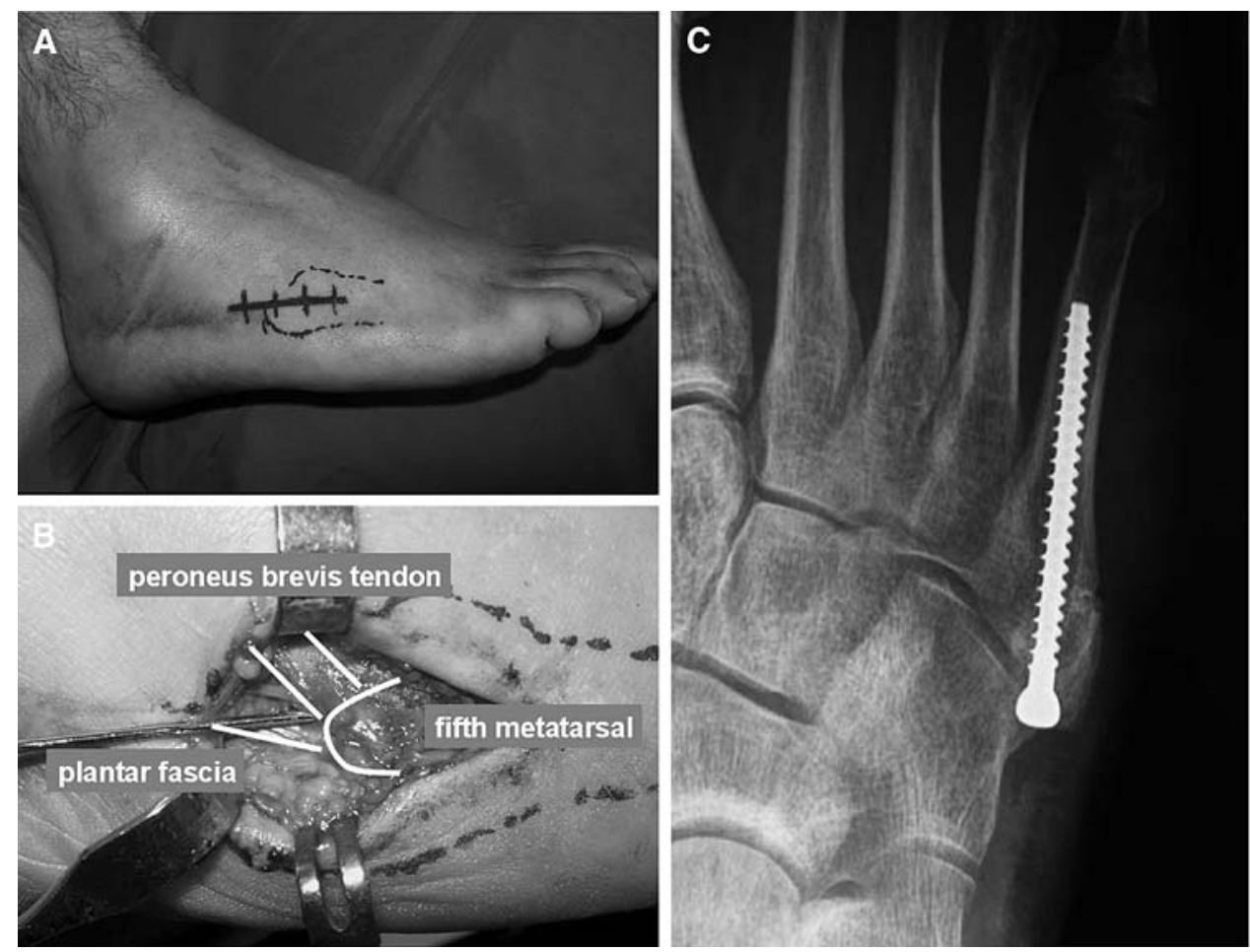

program, return-to-sports time, SAL at follow-up, pain at follow-up (visual analogue scale (VAS): 0-10) and subjective patient satisfaction. Clinical examination contained a complete foot and ankle examination including a foot alignment and arch assessment, ankle instability testing, range of motion measurement (ROM; dorsiflexion/plantar flexion and inversion/eversion), tenderness, and clinical-functional AOFAS midfoot score was registered [6]. Furthermore a complete and distinct neurologic examination of the lower extremity was done to exclude neurologic pathologies [7].

Radiological investigation was performed to document fracture healing at 6 weeks, 3 months, 6 months, 12 months, and final follow-up.

For biomechanical strength measurement maximal isolated isometric force applied for $2 \mathrm{~s}$ were registered. Eversion and inversion strength were measured on both the affected and non-affected foot by using a piezo-electrical element ("Interface" by MFG, Scottsdale, AZ) according to a previous study on foot muscle strength [7] (Fig. 3). To measure the eversion strength (peroneal muscles), the patient was placed in a lateral decubitus position and then asked to maximally evert his or her foot against the plate while the lower leg was fixed to the ground (Fig. 2a). Inversion strength (posterior tibial muscles) was measured in the exactly opposite position maximally inverting his or her foot (Fig. 2b). The mean value was obtained from five sample measurements of the generated strength.

Gait pattern and plantar pressure distribution were analyzed by using the NOVEL dynamic pedobarography

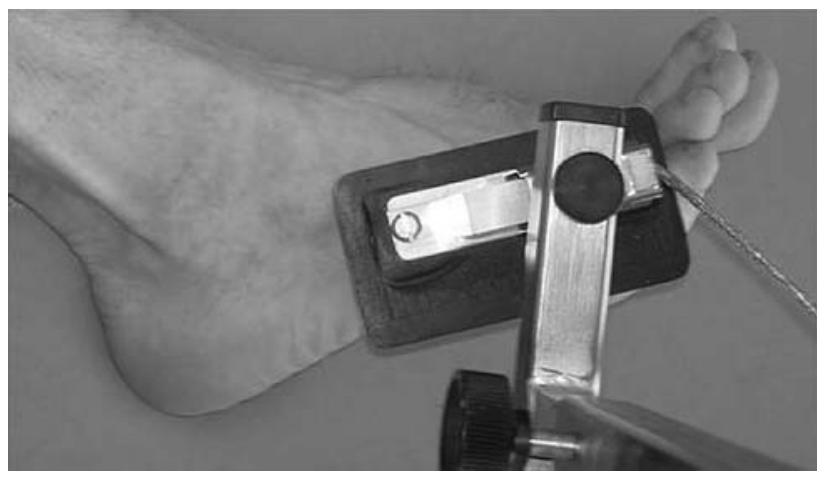

Fig. 3 Muscle strength measurement. The picture shows strength measurement of maximal eversion power (peroneal muscles)

(emed-st, novel GmbH, Munich, Germany). Sensor area measured $240 \times 380 \mathrm{~mm}$, sensor density was $4 / \mathrm{cm}^{2}$ and sample frequency $50 \mathrm{~Hz}$. Measurements consisted of 8 correct trials per foot. For analysis, the trials were averaged. Measured values were maximal ground reaction force, ground contact time, peak pressure, and contact area.

Statistical analysis was performed using the SPSS ${ }^{\circledR}$ software (Version 12.0). Students $t$-test and Pearson correlation were used for calculation, $\alpha$-error was set at 0.05 .

\section{Results}

At follow-up, the overall clinical results were graded excellent in 11 patients, good in three patients, fair in none, and 
poor in none. All patients would in the same situation again agree to undergo the same procedure.

Thirteen patients were completely pain free at follow-up (VAS: 0), one patient complained of local, electrifying pain (VAS 6) that stopped him from restarting with sports activities. Still, he recorded excellent satisfaction with the treatment and would redo the surgery again. Furthermore, one patient complained of subtalar pain that was not associated with the suffered fracture. Two other patients complained of little pain in the morning.

The clinical examination revealed local tenderness or irritation in 4 patients. All of these did not complain of pain but reported of little tenderness maximally while skiing in hard, stiff skiing boots. Two patients complained of electrifying pain while palpating the scar. No local scar problem was seen, one reported from postoperative management. Clinically, normal foot and ankle function (range of motion and gait) was found in 12 patients, one patient suffered a calcaneal fracture and had subtalar osteoarthritis. Neurologically, all included patients had normal, symmetric sensorimotor function.

The average sports activity level (SAL) [5] was 2 [range, 0 (no sports) to 4 (professional and elite sports)] before trauma and also at follow-up. Only the patient who complained of persistent pain could not reach the pre-trauma sports activity level due to pain but also due to time-restriction of his job.

The AOFAS midfoot score for overall pain and functional result was in average: 99 points (range 100-87) with 100 points in 13 cases patients and 87 points in one patient.

Radiologically, all X-rays of postoperative controls were revised and checked for consolidation. Consolidation was found in 9 cases after 6 weeks and in another 3 cases after 12 weeks (range: $39-124$ days), a non-union in one case. In this case, 6 weeks postoperatively, the screw had to be removed because of an infectious wound healing problem. Further treatment was done by foot casting and antibiotics. Follow-up X-ray showed a partial union with a good functional result. In all other cases, no infection or hardware removal was necessary.

The strength measurement showed no significant difference between the treated and the non-affected side (Table 1). The mean peroneal muscle strength was $59 \mathrm{~N}$ on the treated and $64 \mathrm{~N}$ on the contralateral healthy side, corresponding to a ratio of 0.92 between the two legs. $(P=0.53$, $P>0.05)$. Patients' satisfaction $(r=0.0003, P>0.05)$.and sports activities $(r=-0.2, P>0.05)$ did not depend on muscular strength.

The analysis of the dynamic pedobarography results (Table 2) showed no differences in any measurable dimension-for the total foot print (range of ratios: 0.99-1.01). But a slight tendency ( $P$-values $0.19-0.30)$ towards higher ground reaction force, peak pressure and contact area can be seen in the midfoot area, but not in ground contact time. The visual analysis of the gait in the dynamic pedobarography did not evidence any pathology.

\section{Discussion}

This study analyzed intramedullary screw fixation for proximal fifth-metatarsal fractures. An excellent clinical result was registered in the majority of the patients. These find-

Table 1 Biomechanical muscle strength measurement

\begin{tabular}{|c|c|c|c|c|}
\hline & $\begin{array}{l}\text { Operated side } \\
\text { (average } \pm \mathrm{SD})\end{array}$ & $\begin{array}{l}\text { Non-operated side } \\
\text { (average } \pm \mathrm{SD})\end{array}$ & $\begin{array}{l}\text { Ratio operated } \\
\text { verus non-operated side }\end{array}$ & $P$ \\
\hline Eversion strength $(\mathrm{ES} ; \mathrm{N})$ & $59 \pm 8$ & $64 \pm 7$ & 0.92 & 0.53 \\
\hline Inversion strength (IS; N) & $82 \pm 8$ & $101 \pm 8$ & 0.81 & 0.049 \\
\hline Ratio ES/IS & 0.72 & 0.63 & & 0.22 \\
\hline
\end{tabular}

The results show no significant difference for eversion strength and the ES/IS ratio. However, a slight significant difference could be seen for the inversion strength

Table 2 Dynamic pedobarography

The results show no significant difference for maximal ground reaction force, ground contact time, peak pressure and contact area from the affected to the nonaffected contralateral foot

\begin{tabular}{lllcll}
\hline & & $\begin{array}{l}\text { Operated } \\
\text { side }\end{array}$ & $\begin{array}{l}\text { Non-operated } \\
\text { side }\end{array}$ & $\begin{array}{l}\text { Ratio of operated/ } \\
\text { non-operated side }\end{array}$ & $P$ \\
\hline Maximal ground & Total & 990.66 & 996.26 & 0.99 & 0.29 \\
reaction force $(\mathrm{N})$ & Midfoot & 196.04 & 160.06 & 1.22 & 0.19 \\
Ground contact time $(\mathrm{ms})$ & Total & 999.38 & $1,005.61$ & 0.99 & 0.71 \\
& Midfoot & 648.56 & 654.32 & 0.99 & 0.73 \\
Peak pressure $(\mathrm{kPa})$ & Total & 746.53 & 753.81 & 0.99 & 0.87 \\
& Midfoot & 173.18 & 149.14 & 1.16 & 0.27 \\
Contact area $\left(\mathrm{cm}^{2}\right)$ & Total & 142.77 & 141.34 & 1.01 & 0.56 \\
& Midfoot & 30.22 & 28.10 & 1.08 & 0.30 \\
\hline
\end{tabular}


ings were consistent with previous reported data $[2,8,9]$. The radiographic healing was found mainly between 6 and 12 weeks. Similarly, Reese et al. [10] and Portland et al. [9] reported healing times within 6-8 weeks, Fernandez et al. within 8-14 weeks [2]. Although complications like nonunion and persistent pain have been reported [11], this was found to happen mainly in cases in which return to competition was forced. In the presented study, one non-symptomatic, partial healing was found without consequences for the patient's activity.

Classification of proximal fifth-metatarsal fractures is a factor of confusion [12]. Three types of fractures may be distinguished by different anatomical location, pathomechanisms, and type of possible complications [13, 14]: tuberosity avulsion fracture, Jones fracture, proximal diaphyseal fracture (Fig. 1). The most proximal type of fracture is the avulsion fracture of the tuberosity. Being the most distal part of the inversion chain, it is an acute fracture due to a supination trauma of the hind- and mid-foot. Secondary dislocations are frequent due to the tension of the peroneus brevis to the tuberosity fragment. The Jones fracture is a metaphyseal fracture. Although it is presented often as an acute fracture, the pathomechanism shows signs of chronic stress. Located to a critical vascular supply zone [15], delayed- and non-union are frequent. Recommendation for operative treatment is related to the patient's level of activity, most of all for sports active patients and athletes. The most distal type is the proximal diaphyseal fracture. Being a stress fracture, this type of fracture is related closely to sports activities, etiologically often a lateral column overloading as in varus hindfoot malalignment can be found.

Although these different fracture types with different pathomechanisms [13], ISF is the most anatomical way of fixation as it restores biomechanical force transduction from tendon to bone [16] and leads to a good healing in all three fracture types. This way, stable fixation and early weight-bearing is possible including a very-high and fasthealing time. Especially in high demand, young, sports active patients an operative treatment has to be favored as non-union rate in non-operative patients is significantly higher $[2,12,17]$ and secondary treated fractures need a more invasive surgical technique [18]. Alternatively to screw fixation, fixation with a bicortical screw in avulsion type fractures [19], and tension band wiring in small fragment fractures [20] is an option.

Several studies analyzed stress peaks and force directions in the fifth metatarsal [4, 21-25]. Arangio et al. [21] found that maximal stress loading in an oblique direction is consistent with proximal diaphyseal stress fractures. They hypothesized that pronator muscle weakness may predispose to abnormal stress in increasing horizontal load.

Although biomechanics is crucial in this fracture model, no one has controlled the functional outcome after intra- medullary screw fixation by biomechanical measurements. The present study exhibits that pronator muscle strength shows a non-significant ratio of 0.92 from the treated to the contralateral non-affected side. Valderrabano et al. [7] found in posterior tibial tendon reconstructions that side-toside ratios $>0.8$ are not of clinical significance. This finding is consistent with the subjective feeling of the patients who report full restoration of function and strength. A significance was found in the different posterior tibial strength. This might resemble a difference of dominant leg and nondominant leg [26]. It is well known that the different effect of dominant leg and non-dominant leg is more obvious in the tibialis posterior muscle than in the peroneal muscles [26], coincidently the ratio of supination to pronation strength shows no significant difference. This study shows that there is an eversion weakness neither because of a preexisting factor [21] nor because of the fracture or surgical treatment.

Dynamic pedobarography supports these promising functional results furthermore. All parameters-contact time, peak pressure, ground reaction force and contact area-showed side to side ratios between 0.99 and 1.01 . Also sub-analysis of the midfoot area showed no significant differences.

Still, a slight tendency towards a larger contact area, a higher ground reaction force, and an increased peak-pressure can be found. Although these results are not significant, they might be interpreted with the complaint that was found in 4 patients that in hard skiing or ice skating boots, there is little local tenderness. As in all cases, the implants were still in situ, one may expect an improvement after screw removal. But this remains controversial as mainly in high-risk sports like American Football, high re-injury rates are known and some team physicians therefore do not remove the screws. Thus, this is the first study to present a complete restoration of gait function after ISF. However, it is advisable to choose a screw type with a small head and to countersink the head for little soft tissue irritation.

One of the limitations of the study is, that three different types of fractures-avulsion fracture, Jones fracture, and proximal diaphyseal fracture-were analyzed in combination. However, the main focus-clinical and biomechanical aspects after intramedullary screw fixation-is the same for all type of fractures as intramedullary screw fixation is known to be the most secure surgical treatment for all fracture types as also shown in this study. Thus, subgroup analysis did not reveal any significant differences. Another limitation is the size of patient cohort that is too small for wide interpretations. But one has to add, that the findings within the cohort are very consistent so that one should not expect many changings in a bigger cohort. Thirdly, the authors have no group of matched healthy, normal probands. For this, all measurements were compared with the 
results of the healthy, contra-lateral side as it is very usual for in vivo studies of the lower limb. Due to interindividual comparison reasons, no matched control group would fit the contra-lateral side better.

\section{Conclusion}

Intramedullary screw fixation of fifth-metatarsal basal fractures leads to a secure healing and fast return to sports activities. This study could show for the first time that biomechanically a full restoration of gait and motor function could be achieved. The risk of complications is low. No difference in fracture type could be found. Thus, one may conclude that intramedullary screw fixation of proximal fifth-metatarsal fractures is a safe procedure leading to a very-high patient-satisfaction, a high union rate and a fast return to sports time; but a controlled postoperative rehabilitation regime is recommended to prevent failures.

\section{References}

1. Low K, Noblin JD, Browne JE, Barnthouse CD, Scott AR (2004) Jones fractures in the elite football player. J Surg Orthop Adv 13(3):156-160

2. Fernandez Fairen M, Guillen J, Busto JM, Roura J (1999) Fractures of the fifth metatarsal in basketball players. Knee Surg Sports Trauma Arthrosc 7:373-377

3. Jones R (1902) Fracture of the base of the fifth metatarsal by indirect violence. Ann Surg 35:697-702

4. Horst F, Gilbert BJ, Glisson RR, Nunley JA (2004) Torque resistance after fixation of jones fractures with intramedullary screws. Foot Ankle Int 25(12):914-919

5. Valderrabano V, Pagenstert G, Horisberger M, Knupp M, Hintermann B (2006) Sports and recreation activity of ankle arthritis patients before and after total ankle replacement. Am J Sports Med 34(6):993-999

6. Kitaoka HB, Alexander IJ, Adelaar RS, Nunley JA, Myerson MS, Sanders M (1994) Clinical rating systems for the ankle-hindfoot, midfoot, hallux, and lesser toes. Foot Ankle Int 15(7):349-353

7. Valderrabano V, Hintermann B, Wischer T, Fuhr P, Dick W (2004) Recovery of the posterior tibial muscle after late reconstruction following tendon rupture. Foot Ankle Int 25:85-95

8. Porter DA, Duncan M, Meyer SJF (2005) Fifth metatarsal jones fracture fixation with a $4.5-\mathrm{mm}$ cannulated stainless stell screw in the competitive and recreational athlete. Am J Sports Med 33(5):726-733

9. Portland G, Kelikian A, Kodros S (2003) Acute surgical management of Jones' fracture. Foot Ankle Int 24(11):829-833

10. Reese K, Litsky A, Kaeding CC, Pedroza A, Shah N (2004) Cannulated screw fixation of Jones fractures. Am J Sports Med 32(7):1736-1742

11. Larson CM, Almekinders LC, Taft TN, Garrett WE (2002) Intramedullary screw fixation of Jones fractures: analysis of failure. Am J Sports Med 30(1):55-60

12. Clapper MF, O'Brien TJ, Lyons PM (1995) Fractures of the fifth metatarsal: analysis of a fracture registry. CORR 315:238-241

13. Lawrence SJ, Botte MJ (1993) Jones fractures and related fractures of the fifth metatarsal. Foot Ankle Int 14:358-364

14. TB Dameron Jr (1995) Fractures of the proximal fifth metatarsal: selecting the best treatment option. J Am Acad Orthop Surg $3(2): 110-114$

15. Smith JW, Arnoczky SP, Hersh A (1992) The intraosseus blood supply of the fifth metatarsal: implications for proximal fracture healing. Foot Ankle Int 13(3):143-152

16. Bong MR, Kummer FJ, Egol KA (2007) Intramedullary nailing of the lower extremity: biomechanics and biology. J Am Acad Orthop Surg 15(2):97-106

17. Josefsson PO, Karlsson M, Redlund-Johnell I, Wendeberg B (1994) Jones fracture. Surgical versus nonsurgical treatment. CORR 299:252-255

18. Lawrence SJ (2004) Technique tip: local bone grafting technique for Jones fracture management with intramedullary screw fixation. Foot Ankle Int 25(12):920-921

19. Husain ZS, DeFronzo DJ (2000) Relative stability of tension band versus two-cortes screw fixation for treating fifth metatarsal base avulsion fractures. J Foot Ankle Surg 39(2):89-95

20. Sarimo J, Rantanen J, Orava S, Alanen J (2006) Tension-band wiring for fractures of the fifth metatarsal located in the junction of the proximal metaphysis and diaphysis. Am J Sports Med 34(3):476480

21. Arangio GA, Xiao D, Salathe EP (1997) Biomechanical study of stress in the fifth metatarsal. Clin Biomech 12(3):160-164

22. Donahue SW, Sharkey NA (1999) Stance phase of gait: implications for stress fractures. JBJS Am 81(9):1236-1244

23. Giordano AR, Ferkel RD (2004) Strength analysis of intraosseous wire fixation for avulsion fractures of the fifth metatarsal base. J Foot Ankle Surg 43(4):225-230

24. Moshirfar A, Campbell JT, Molloy S, Jasper LE, Belkoff SM (2003) Fifth metatarsal tuberosity fracture fixation: a biomechanical study. Foot Ankle Int 24(8):630-633

25. Vertullo CJ, Glisson RR, Nunley JA (2004) Torsional strains in the proximal fifth metatarsal: implications for Jones and stress fracture management. Foot Ankle Int 25(9):651-656

26. Valderrabano V, von Tscharner V, Nigg B, Hintermann B, Goepfert B, Fung TS, Frank CB, Herzog W (2006) Lower leg muscle atrophy in ankle osteoarthritis. J Orthop Res 24(12):2159-2169 